\title{
Pratiques de protection des cultures en agroécosystèmes tropicaux et risques de maladies humaines et animales d'origine bactérienne
}

\author{
Alain Ratnadass ${ }^{1,2, *}$ (i) et Péninna Deberdt ${ }^{2,3}$ \\ ${ }^{1}$ CIRAD, UPR HortSys, Saint-Pierre F-97455, La Réunion, France \\ ${ }^{2}$ HortSys, Univ. Montpellier, CIRAD, Montpellier, France \\ ${ }^{3}$ CIRAD, UPR HortSys, F-34398 Montpellier, France
}

\begin{abstract}
Résumé - Une revue bibliographique récente montre que les pratiques de protection agroécologique des cultures (PAEC) contre les ravageurs, pathogènes et adventices, réduisent généralement les risques zoonotiques viraux en santé publique et vétérinaire, alors que les pratiques conventionnelles (à base agrochimique) tendent à les augmenter. Nous avons analysé la littérature scientifique pour déterminer si ces conclusions pouvaient être élargies aux infections bactériennes, avec focus sur les agroécosystèmes tropicaux. En santé publique et vétérinaire, les principales infections bactériennes impactées par des pratiques de protection des cultures sont celles ayant pour réservoirs des rongeurs (avec arthropodes vecteurs, comme la peste, ou pas, comme la leptospirose), et les maladies à entérobactéries (e.g., Salmonella) et autres bactéries opportunistes (e.g., Pseudomonas). La problématique d'antibiorésistance en santé publique est peu affectée par les pratiques de protection des cultures conventionnelles. Des pratiques relevant de la substitution aux intrants de synthèse (variétés résistantes aux attaques de rongeurs ou à la colonisation par des entérobactéries; pesticides minéraux ; solarisation) peuvent se traduire par une réduction plus importante du risque sanitaire que certaines pratiques relevant de la reconception des agroécosystèmes (push-pull ; intégration agriculture-élevage). Toutefois, vu ses effets vertueux en termes de réduction des pollutions, conservation de la biodiversité et résilience climatique, la PAEC, basée sur la reconception, contribue, à des échelles spatiotemporelles supérieures, à la réduction du risque sanitaire lié aux infections bactériennes. Les impacts des pratiques de protection des cultures, basées sur l'amélioration de l'efficience des pratiques conventionnelles, sur la substitution ou sur la reconception, sur les risques d'infection bactérienne en santé publique et vétérinaire, sont sensiblement différents de ceux observés avec les risques zoonotiques viraux. Des recherches sont encore nécessaires pour concilier, dans une perspective "Une seule santé», les trois types de pratiques, afin de réduire les risques sanitaires liés aux maladies infectieuses autant à bactéries qu'à virus, parasites (protozoaires et vers) ou champignons.
\end{abstract}

Mots clés : antibiotiques / cuivre / rotation culturale / paillage / rhizobactéries

\begin{abstract}
Crop protection practices in tropical agroecosystems and risks of human and animal diseases of bacterial origin. A recent literature survey has shown that Agroecological Crop Protection (ACP) practices against pests, pathogens and weeds, generally alleviate viral zoonotic risks in public and veterinary health, contrary to conventional (agrochemistry-based) practices, which tend to increase the same. We analyzed the scientific literature to determine whether these conclusions could be extended to bacterial infections, with a focus on tropical agroecosystems. In public and veterinary health, the main bacterial infections being impacted by crop protection practices are those with rodent reservoirs (either with arthropodan vector, e.g., plague, or not, e.g., leptospirosis), and diseases caused by enterobacteria (e.g., Salmonella) and other opportunistic bacteria (e.g., Pseudomonas). The antimicrobial resistance issue in public health is barely affected by conventional crop protection practices. Some practices based on substitution to synthetic inputs (crop cultivars resistant to rodent attacks or to colonization by enterobacteria; mineral pesticides; solarization) are more conducive to sanitary risk alleviation than some redesign-based
\end{abstract}

\footnotetext{
*Auteur de correspondance : alain.ratnadass@cirad.fr
} 
ones (push-pull; crop-livestock integration). However, given its virtuous impacts in terms of pollution alleviation, biodiversity conservation, and climatic resilience, ACP contributes, at higher spatiotemporal scales, to the reduction of bacterial infection-based sanitary risks. Impacts in public and veterinary health of crop protection practices, either based on the improvement of the efficiency of conventional practices, on substitution, or on tropical agroecosystems redesign, on bacterial infection risks, still differ significantly from those observed on viral zoonotic risks. Research is still needed in view of reconciling, in a "One health" perspective, the three types of practices, to reduce sanitary risks relating to infectious diseases, whether of bacterial, viral, parasitic (protozoan or worm) or fungal origin.

Keywords: antibiotics / copper / crop rotation / mulching / rhizobacteria

\section{Introduction}

La protection agroécologique des cultures (PAEC) contre les bioagresseurs (ravageurs, pathogènes et adventices) favorise le fonctionnement écologique des agroécosystèmes, en optimisant directement ou indirectement les interactions entre les communautés vivantes (végétales, animales et microbiennes) souterraines et aériennes (Deguine et al., 2016). Les pratiques de PAEC réduisent généralement les risques de zoonoses virales (Polack et al., 2015), comme les infections à virus Nipah ou West Nile, alors que celles de protection des cultures conventionnelles (à base agrochimique) tendent à les augmenter (Ratnadass et Deguine, 2021).

En effet, les insecticides de synthèse et rodenticides anticoagulants, visant les ravageurs des cultures, sélectionnent des populations résistantes respectivement chez les arthropodes vecteurs de zoonoses virales (e.g., moustiques vecteurs de virus West Nile) qui y sont indirectement exposés, et chez les rongeurs ciblés, souvent aussi réservoirs de zoonoses virales (e.g., Hantavirus) (Ratnadass et Deguine, 2021). A contrario, les pratiques de PAEC diluent généralement les risques de transmission de zoonoses virales, via la diversification végétale dans les agroécosystèmes et la biodiversité animale induite, et favorisent les régulations naturelles des populations de vertébrés réservoirs de zoonoses virales. Elles réduisent aussi indirectement ces risques par atténuation du réchauffement climatique (e.g., PAEC en systèmes agroforestiers et d'agriculture de conservation) et par ralentissement de l'expansion géographique d'arthropodes vecteurs (Ratnadass et Deguine, 2021).

On peut se demander ce qu'il en est pour les autres organismes pathogènes en santé publique et vétérinaire, notamment les bactéries. Nous effectuons donc ici une revue de la littérature scientifique concernant les effets des pratiques de protection des cultures contre les bioagresseurs, sur les infections bactériennes en santé publique et vétérinaire, avec focus sur les agroécosystèmes tropicaux. Nous nous appuyons pour cela en partie sur les revues bibliographiques récentes de Mendes et al. (2013), Sundin et al. (2016) et Lenzi et al. (2021), respectivement sur le statut des microorganismes rhizosphériques, la gestion des maladies bactériennes des plantes, et l'effet des pratiques agronomiques sur la contamination des cultures maraîchères par les entérobactéries pathogènes de l'homme.

Nous suivons le même cadre conceptuel que Ratnadass et Deguine (2021), i.e., la typologie E-S-R (Hill et MacRae, 1996), qui recouvre respectivement: i) l'amélioration de l'Efficience (E) des pratiques conventionnelles de protection des cultures fondées sur l'agrochimie; ii) la Substitution (S) de ces pratiques par des méthodes génétiques, culturales, physiques/mécaniques ou par recours à des produits de biocontrôle (macroorganismes et microorganismes auxiliaires, médiateurs chimiques et substances naturelles d'origine végétale, animale ou minérale [Herth, 2011]); et iii) la Reconception $(\mathrm{R})$ de l'agroécosystème pour le rendre résilient aux bioagresseurs (avec recours essentiellement à la lutte biologique par conservation et gestion des habitats).

\section{Amélioration de l'efficience}

\subsection{Insecticides de synthèse}

Il n'y a pas, à notre connaissance, d'exemple où l'application d'insecticides de synthèse pour la protection des cultures réduit l'incidence d'infections bactériennes en santé publique et vétérinaire tropicale. En effet, les insecticides de synthèse utilisés contre les puces (e.g., Xenopsylla cheopis) vectrices du bacille de la peste (Yersinia pestis) le sont dans une optique de santé publique, pas de protection des cultures.

En revanche, des traitements biocides visant des arthropodes ravageurs de cultures peuvent (au moins potentiellement) induire des résistances aux insecticides de synthèse chez ces puces (Rugalema et Mnyone, 2020) et aux acaricides chez les tiques Rhipicephalus/Boophilus vectrices d'anaplasmoses (Gondard et al., 2017 ; Kumar et al., 2020). Ces insecticides de synthèse ou acaricides favorisent ainsi indirectement les infections bactériennes, comme pour les moustiques vecteurs de zoonoses virales (Ratnadass et Deguine, 2021).

\subsection{Rodenticides}

Pour lutter contre les rongeurs ravageurs de cultures et de récoltes stockées, les rodenticides, notamment les rodenticides anticoagulants, se montrent d'abord efficaces à réduire les populations de ces réservoirs (e.g., Rattus spp. pour le bacille de la peste [Rahelinirina et al., 2021], les rickettsies du typhus murin [Rickettsia typhi: Rakotonanahary et al., 2017] ou les spirochètes de la leptospirose [Leptospira spp. : Marquez et al., 2019]). Mais des résistances à ces rodenticides apparaissent ensuite (Ratnadass et Deguine, 2021).

La lutte létale contre ces nuisibles doit aussi tenir compte du risque de transmission supérieur pour la peste (ou le typhus) que pour les zoonoses virales, car étant le fait de puces qui, en quittant les cadavres, recherchent activement d'autres hôtes (Miarinjara et al., 2019). Cela s'ajoute au risque sanitaire pour l'homme et les animaux domestiques carnivores, par la manipulation sans précaution des cadavres de rats infectés. Pour la leptospirose, les législations européenne et française 
limitent au seul champ l'utilisation des rodenticides anticoagulants, et à deux périodes de culture de la canne à sucre dans l'année à La Réunion (Mansuy et Fontaine, 2014). En Asie du Sud-Est, c'est surtout durant les travaux champêtres (notamment en rizières et champs de canne à sucre) que les producteurs sont infectés par les spirochètes de la leptospirose (Tangkanakul et al., 2005), quoique pas uniquement pendant des opérations de protection des cultures.

\subsection{Antibiotiques}

L'utilisation massive d'antibiotiques en médecine vétérinaire (voire zootechnie) peut induire une antibiorésistance chez des bactéries de la flore intestinale du bétail, dont certaines sont pathogènes de l'homme, d'où un risque sanitaire à utiliser des engrais d'origine animale (Jechalke et al., 2014). Il n'y a en revanche pas de cas documenté où l'application en agriculture d'antibiotiques contre des bactéries phytopathogènes entraînerait des antibiorésistances chez des bactéries pathogènes en santé publique et vétérinaire (McManus et al., 2002 ; Sundin et al., 2016). Toutefois, c'est bien la gravité de la crise occasionnée par les antibiorésistances généralisées chez l'homme qui limite l'utilisation d'antibiotiques en protection des cultures, la cantonnant aux cultures horticoles à forte valeur (McManus et al., 2002).

Cela exclut a priori une contribution importante de l'injection d'antibiotiques dans le tronc d'arbres fruitiers à l'antibiorésistance de bactéries pathogènes à ces antibiotiques (notamment la streptomycine) en santé publique et vétérinaire (Stockwell et Duffy, 2012 ; McManus, 2014). En revanche, les pulvérisations foliaires d'antibiotiques à grande échelle pour lutter contre le Greening (Huanglongbing) des agrumes, causé par Candidatus Liberibacter sp., comme depuis peu en Floride (McKenna, 2019), ou l'application inconsidérée d'antibiotiques à usage spécifiquement médical comme en Thaïlande (Chanvatik et al., 2019), posent question.

\section{Substitution}

\subsection{Variétés résistantes}

Les variétés de plantes cultivées résistantes à des bioagresseurs spécifiques étant des alternatives pour remplacer des composantes peu efficaces du système de culture, leur déploiement relève bien d'une approche « $\mathrm{S} »$. C'est pour la canne à sucre, particulièrement sujette aux attaques de rongeurs, le cas des variétés à écorce dure (Tobin, 1994) et port érigé comme la «canne cannelle» (B 82.333) en Martinique (Quénéhervé et al., 2005), ou la «canne paille» R 570 (résistante au foreur de tiges Chilo sacchariphagus à la Réunion: Nibouche et Tibère, 2010), moins attaquées par les rongeurs que la «canne roseau » $\mathrm{R} 59.92$. Cette réduction des attaques réduit aussi les risques d'infection au champ par la leptospirose.

Il y a aussi des différences variétales dans la capacité des cultures maraîchères à héberger des entérobactéries pathogènes pour l'homme (Lenzi et al., 2021). En cultivant des variétés de légumes résistantes aux champignons et bactéries phytopathogènes qui favorisent la colonisation de légumes par des entérobactéries, on réduit le risque d'infections bactériennes. C'est le cas des variétés de concombre Marketmore 97 ou de tomate «Sun Gold» et «Snow White» vis-à-vis de Salmonella enterica (Lenzi et al., 2021).

\subsection{Pesticides minéraux et produits de biocontrôle}

Sur tomate, l'application foliaire de cuivre réduit les populations d'entérobactéries, e.g., Salmonella et Paenibacillus (Lenzi et al., 2021). La résistance croisée des entérocoques à l'érythromycine est quant à elle attribuée au cuivre utilisé comme additif en alimentation des volailles et des porcs, plutôt qu'à l'utilisation de produits cuivrés en protection des cultures (Sundin et al., 2016).

Plusieurs souches de Burkholderia, homologuées comme actives sur des champignons du sol phytopathogènes, ont été retirées car responsables de maladies humaines (Eberl et Vandamme, 2016). Egalement, plusieurs bactéries efficaces en traitement post-récolte contre l'anthracnose de la banane (e.g., Enterobactercloacae, Serratia marcescens et Stenotrophomonas maltophilia) sont pathogènes de l'homme, notamment des individus immunodéprimés (Damasceno et al., 2019).

De même, alors que Pseudomonas fluorescens est largement mentionné dans la littérature comme agent de biocontrôle (Couillerot et al., 2009), Pseudomonas aeruginosa, qui a également un effet protecteur des plantes, en particulier de la tomate contre Botrytis cinerea (Mendes et al., 2013), présente un réel risque infectieux pour l'homme lors d'opérations d'isolement, de purification et de multiplication pour élaborer des produits de biocontrôle. Exemple-type des bactéries opportunistes nosocomiales, P. aeruginosa est peu ou pas virulente chez l'individu sain, mais peut s'avérer redoutable chez les sujets immunodéprimés (Bodey et al., 1983). En revanche, divers extraits végétaux ont un effet bactériostatique à la fois sur Ralstonia solanacearum, responsable du flétrissement bactérien des Solanacées (Fig. 1) et sur P.aeruginosa (Rasamiravaka et al., 2018).

\subsection{Paillage et solarisation}

Par la solarisation (substitut à la fumigation), le paillage plastique contrôle les nématodes et autres pathogènes telluriques, mais décontamine aussi le sol des entérobactéries (Escherichia coli et Salmonella) (Lenzi et al., 2021). Il est aussi utilisé en maraîchage pour contrôler les adventices. En empêchant le contact direct des organes végétaux aériens avec le sol, il réduit l'incidence de certaines maladies des plantes et leur contamination par des entérobactéries.

En revanche, par rapport au sol nu, il favorise la persistance des entérobactéries dans le sol et leur dispersion par les éclaboussures liées à la pluie ou l'irrigation. Par ailleurs, les populations d'E. coli se maintiennent mieux dans les laitues avec paillage (plastique, biodégradable ou végétal) que dans celles sur sol nu (Lenzi et al., 2021).

\subsection{Autres pratiques culturales}

La gestion des adventices, avec herbicides ou manuelle, réduit dans les champs les populations de rongeurs (auxquels l'enherbement sert de refuge et de source de nourriture 


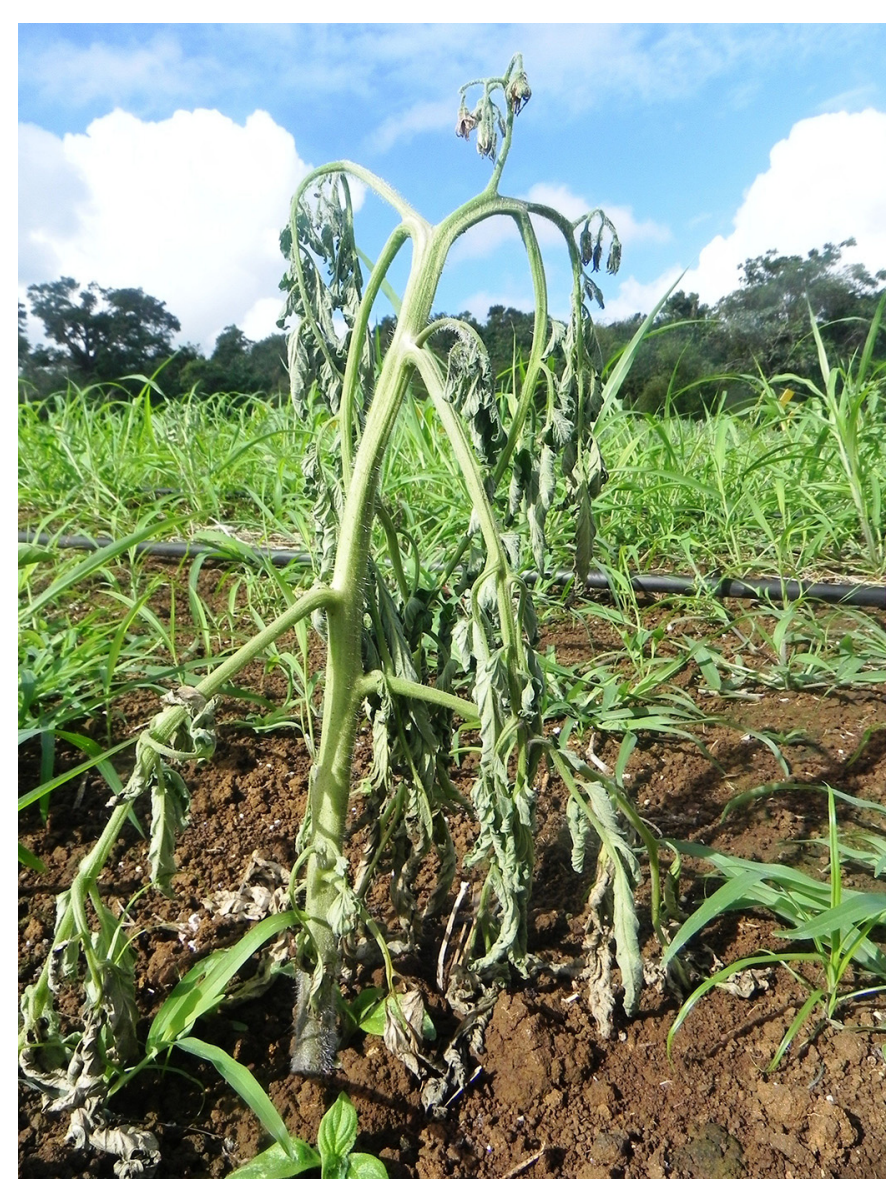

Fig. 1. Plant de tomate avec symptôme typique de flétrissement bactérien dû à Ralstonia solanacearum, en plein champ en Martinique.

Fig. 1. Tomato plant with a typical symptom of bacterial wilt disease caused by Ralstonia solanacearum under field conditions in Martinique.

alternative via les graines d'adventices: Htwe et al., 2019) et les risques d'infection bactérienne associés. Le brûlis (Massawe et al., 2005), comme moyen de désherbage et de destruction de l'habitat des rongeurs, a cependant pour effet pervers de chasser les rats vers les villages, augmentant les risques de transmission d'infections bactériennes aux humains et animaux domestiques.

Les fumiers peuvent être appliqués en partie pour réduire les populations de nématodes phytoparasites, quoique dans une moindre mesure que les composts végétaux (Litterick et al., 2004). Ils peuvent toutefois aussi transmettre des pathogènes humains (notamment $E$. coli) ou des gènes d'antibiorésistance (Xie et al., 2018 ; Yoshizawa et al., 2020).

\section{Reconception}

\subsection{Plantes de service en rotation}

Outre les microorganismes bénéfiques pour la croissance des plantes et les phytopathogènes, on trouve dans le microbiome des rhizosphères des bactéries opportunistes pathogènes pour l'homme (des genres Burkholderia,
Enterobacter, Herbaspirillum, Ochrobactrum, Pseudomonas, Ralstonia, Staphylococcus et Stenotrophomonas) (Mendes et al., 2013). Or, celles-ci sont favorisées si on cultive, en rotation avec la culture principale, des plantes « de service» les hébergeant dans leur rhizosphère.

Ainsi, la culture en précédent de certaines variétés de blé rend les sols suppressifs par rapport à Rhizoctonia solani, qui affecte négativement la croissance de jeunes plants de pommiers (Gu et Mazzola, 2003). Or, cet effet suppressif est dû à la compétition avec Pseudomonas putida, potentiellement pathogène pour l'homme (Fernández et al., 2015).

\subsection{Push-Pull}

Cette technique implique des bordures de plantes pièges, dont certaines assez touffues, et des plantes répulsives souvent à fort pouvoir couvrant, associées au céréales (Khan et al., 2011). Ces bordures et plantes associées peuvent servir d'habitats aux rongeurs ravageurs et potentiellement réservoirs d'infections bactériennes, à l'instar de l'enherbement spontané (cf. Sect. 3.4). Ainsi, les haies de Vetiver (Vetiveria zizanoides), plante-piège pour des foreurs des tiges (van den Berg, 2006), sont considérées par certains comme refuges pour les rongeurs, et par d'autres au contraire comme répulsives des rongeurs autant que des serpents (Grimshaw et Faiz, 1995).

\subsection{Lutte biologique par conservation et intégration agriculture-élevage}

À l'instar des bordures de plantes-pièges ( $c f$. Sect. 4.2), les plantes à fleurs de l'enherbement spontané des parcelles cultivées ou des haies ou bordures enherbées, utilisées en lutte biologique par conservation, peuvent servir d'habitats à diverses espèces de rongeurs, dont certains sont réservoirs d'infections bactériennes comme la peste (Rahelinirina et Duplantier, 1997; Rahelinirina et al., 2010) ou la tularémie causée par Francisella tularensis (Rodríguez-Pastor et al., 2017), ou des infections bactériennes à entérobactéries. Toutefois, ces infrastructures écologiques n'augmentent pas systématiquement le risque de dégâts aux cultures, ou de contamination par des pathogènes, à témoin la non-aggravation des cas de Salmonella et E. coli en vergers de noyers et parcelles de tomate avec haies en Californie (Sellers et al., 2018).

Les nichoirs et perchoirs pour les chouettes contribuent bien à combattre les rongeurs en tant que réservoirs d'infections bactériennes comme de zoonoses virales (Ratnadass et Deguine, 2021). En revanche, la stratégie de "paysages de peur» par l'attraction de rapaces pour effrayer les oiseaux granivores ou frugivores dans des espaces dégagés, via l'exposition de carcasses d'animaux (Leggett, 2012), pose le problème de leur manipulation et ainsi d'exposition à des infections bactériennes.

L'introduction de volailles dans les vergers permet d'y gérer l'enherbement (e.g., avec les oies) ou les insectes ravageurs (e.g., avec les poules) (Ratnadass et Deguine, 2021). Toutefois, cela peut aussi accroître les risques de transmission d'entérobactéries ( $c f$. Sect. 3.4 et Pires et al., 2019; Theofel et al., 2020). 


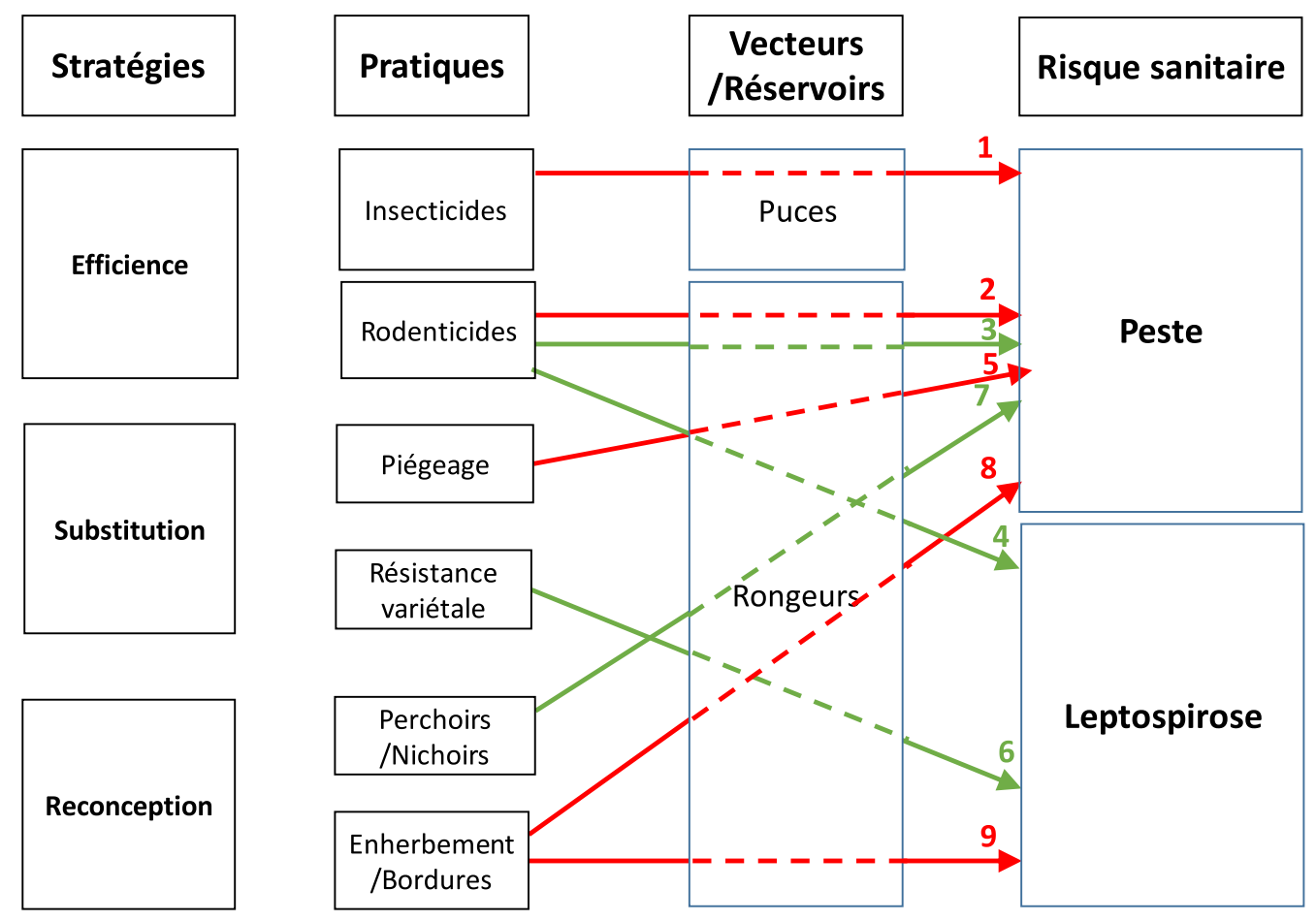

Fig. 2. Impact de pratiques de protection des cultures relevant de stratégies d'efficience, de substitution ou de reconception (Hill et MacRae, 1996) sur les risques sanitaires bactériens pour l'homme: cas de la peste et de la leptospirose.

Flèche rouge: effet négatif (augmentation du risque sanitaire); Flèche verte: effet positif (réduction du risque sanitaire. La numérotation renvoie aux références bibliographiques explicitant les processus.

1. Rugalema et Mnyone (2020); 2. Andru et al. (2013); 3. Rahelinirina et al. (2021); 4. Marquez et al. (2019); 5. Meerburg et al. (2009); 6. Quénéhervé et al. (2005) ; 7. Bessou et al. (2017); 8 et 9. Rahelinirina et Duplantier (1997); Rahelinirina et al. (2010); Htwe et al. (2019); Massawe et al. (2005).

Fig. 2. Impact of Efficiency-, Substitution- or Redesign-based (Hill and MacRae, 1996) crop protection practices on bacterial sanitary risks for humans: case of plague and leptospirosis.

Red arrow: negative impact (increased sanitary risk); Green arrow: positive impact (reduced sanitary risk). Numbering refers to literature references detailing processes.

\section{Discussion}

Les résultats de notre analyse sont synthétisés dans les figures 2 et 3 pour les deux principaux groupes d'infections bactériennes couverts, respectivement celles avec des rongeurs comme réservoirs, et celles à entérobactéries et autres bactéries opportunistes. Les conclusions tirées diffèrent pour partie de celles concernant les zoonoses virales (Ratnadass et Deguine, 2021).

Ainsi, on a moins d'exemples d'effets de pratiques «Efficience» sur le risque sanitaire lié aux infections bactériennes, et la balance penche plutôt en faveur d'un effet réducteur du risque par ces pratiques au final. Cela essentiellement via la réduction du risque de leptospirose par utilisation de rodenticides contre les rongeurs ravageurs des cultures, sous réserve de mesures de précaution comme la non manipulation des cadavres à mains nues. Cela aussi en partie parce qu'il y a moins d'arthropodes vecteurs d'infections bactériennes que de vecteurs de zoonoses virales, à pouvoir être affectés par des traitements insecticides ou acaricides sur cultures. Enfin, si l'antibiorésistance est une contrainte majeure, centrale dans la problématique «Une seule santé », elle est peu alimentée par les pratiques de protection des cultures proprement dites.
En revanche, l'introduction d'animaux d'élevage pour gérer des bioagresseurs dans les agroécosystèmes (stratégie «Reconception»), ou l'utilisation de leurs fumiers à fins (au moins en partie) de protection des cultures (stratégie «Substitution»), peut, outre les risques de contamination directe avec entérobactéries (supérieurs à ceux dus aux entérovirus: Bicudo et Goyal, 2003), favoriser des antibiorésistances, y compris chez des bactéries endophytes de plantes consommées par l'homme. Cependant, dans les exploitations d'agriculture-élevage conduites en agriculture biologique, les antibiotiques vétérinaires sont proscrits, en faveur de «phytobiotiques » (e.g., huiles essentielles) moins sujets à antibiorésistance (Diaz-Sanchez et al., 2015). Il en est de même dans les exploitations engagées dans une démarche de transition agroécologique. Le risque de contamination par entérobactéries est aussi réduit si les fumiers sont compostés à des températures suffisamment élevées.

Une autre différence tient à ce que des bactéries ayant pour hôtes des plantes cultivées, ou hébergées dans leur rhizosphère, peuvent être pathogènes pour l'homme (ce qui est moins fréquent pour les entérovirus). La résistance variétale des cultures à la colonisation par ces bactéries, ou aux microorganismes phytopathogènes la favorisant, est donc une 


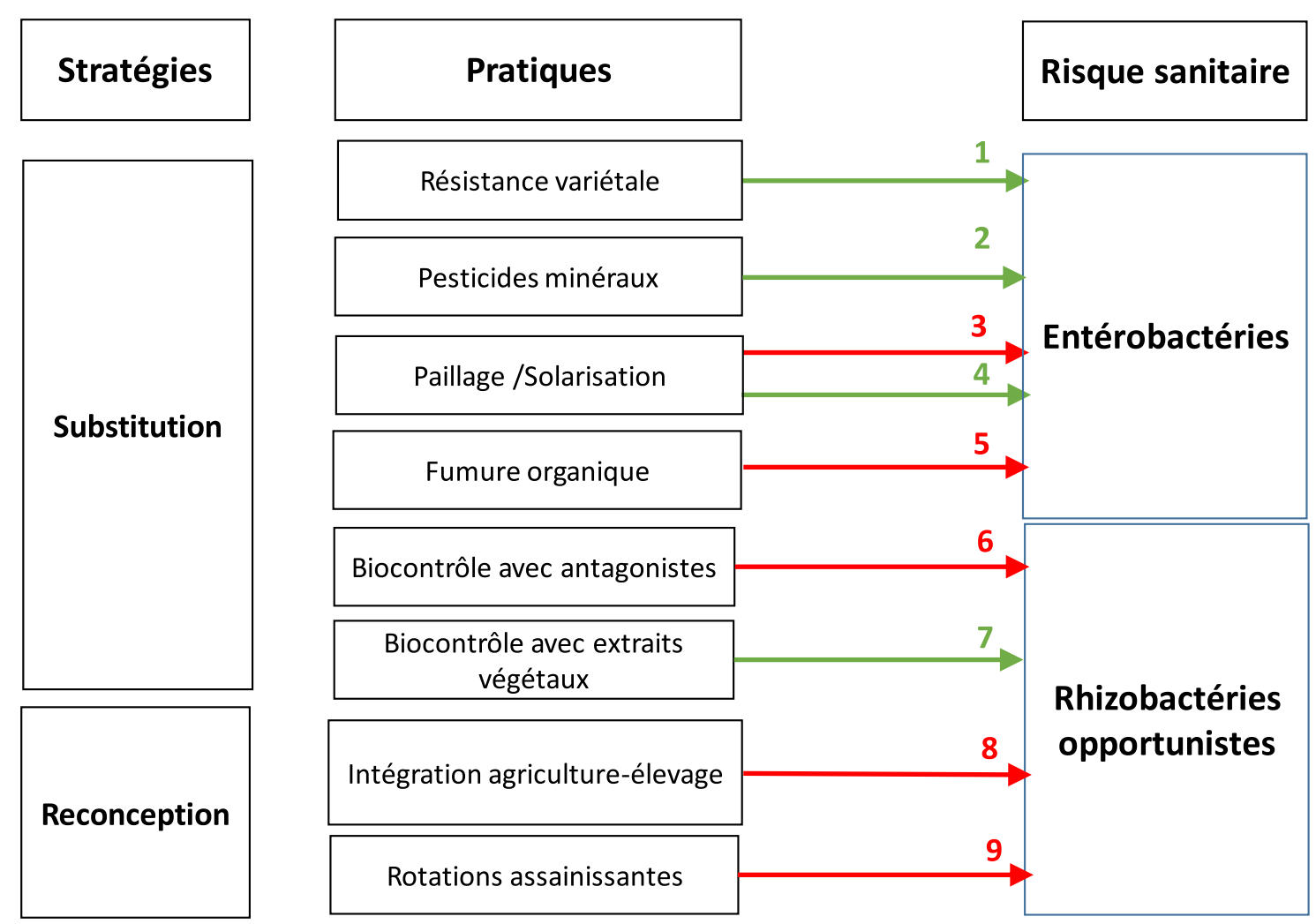

Fig. 3. Impact de pratiques de protection des cultures relevant de stratégies de substitution ou reconception (Hill et MacRae, 1996) sur les risques sanitaires bactériens pour l'homme: cas des infections à entérobactéries et rhizobactéries pathogènes opportunistes.

Flèche rouge: effet négatif (augmentation du risque sanitaire); Flèche verte: effet positif (réduction du risque sanitaire. La numérotation renvoie aux références bibliographiques explicitant les processus.

1. Callahan et Micallef (2019); Henriquez et al. (2020); 2. Ottesen et al. (2015); 3. Wu et al. (2009); 4. Xu et al. (2016); 5. Xie et al. (2018); Yoshizawa et al. (2020); 6. Eberl et Vandamme (2016); Damasceno et al. (2019); 7. Rasamiravaka et al. (2018); 8. Theofel et al. (2020); Pires et al. (2019); 9. Gu et Mazzola (2003); Fernández et al. (2015).

Fig. 3. Impact of Substitution- or Redesign-based (Hill and MacRae, 1996) crop protection practices on bacterial sanitary risks for humans: case of enterobacteria and opportunistic pathogenic rhizobacteria.

Red arrow: negative impact (increased sanitary risk); Green arrow: positive impact (reduced sanitary risk). Numbering refers to literature references detailing processes.

voie pertinente pour réduire l'incidence d'infections bactériennes chez l'homme. Il en est de même d'autres pratiques de type «Substitution» (e.g., pesticides minéraux à base de cuivre; solarisation).

Par ailleurs, certaines modalités de déploiement spatiotemporel de diversité végétale relevant de la PAEC (stratégie «Reconception») contre des microorganismes phytopathogènes (e.g., rotations assainissantes) ou des arthropodes ravageurs (e.g., push-pull ou lutte biologique par conservation), favorisent des rhizobactéries pathogènes opportunistes de l'homme, ou des rongeurs ravageurs des cultures et réservoirs d'infections bactériennes. Ainsi, en Martinique, l'espèce Allium fistulosum (appelée «oignon péyi») est utilisée en rotations ou associations culturales dans les systèmes maraîchers pour lutter contre le flétrissement bactérien de la tomate dû à $R$. solanacearum (Fig. 1). Or, des bactéries, isolées dans la rhizosphère d'A. fistulosum, se sont révélées antagonistes de R. solanacearum (in vitro), exprimant un effet protecteur sur la tomate par réduction drastique de l'incidence du flétrissement bactérien en conditions in vivo (Deberdt, données non publiées). Leur caractérisation moléculaire (séquençage du gène de l'ARNr 16 S) a révélé leur appartenance à l'espèce P.aeruginosa (Deberdt, données non publiées), qui présente un risque infectieux potentiel pour l'homme (cf. Sect. 3.2).

L'enjeu de bien-être animal n'est pas aussi prégnant pour les infections bactériennes que pour les zoonoses virales, d'une part vu la moindre importance des chauves-souris (notamment frugivores) dans leur épidémiologie, et d'autre part parce que les infections bactériennes sont moins responsables d'épidémies en élevages intensifs (notamment de volailles et de porcs). Concernant la conservation de la biodiversité (au cour de la démarche PAEC), les infections bactériennes sont, à l'instar des zoonoses virales, notamment quand elles impliquent des rongeurs réservoirs (e.g., la leptospirose et la peste), sujettes à dilution par conservation ou augmentation de la diversité spécifique des hôtes (Derne et al., 2011 ; McCauley et al., 2015). C'est aussi le cas de la maladie de Lyme causée par Borrelia burgdorferi (LoGiudice et al., 2003), qui n'a toutefois pas été prise en compte dans cette revue car en plus que de ne concerner quasiment que les zones tempérées, l’impact bénéfique de son rongeur réservoir (Peromyscus 
leucopus), par la consommation de graines d'adventices et d'insectes ravageurs, compense les dégâts qu'il peut occasionner aux cultures, rendant caduques d'éventuelles mesures de protection des cultures à son encontre (Howell et al., 2017).

\section{Conclusion}

Contrairement au risque de zoonoses virales, les pratiques de type «Reconception» ne sont pas forcément plus performantes que celles de types «Efficience» et «Substitution» pour réduire le risque d'infections bactériennes. Toutefois, dans une perspective «Une seule santé», les bénéfices de la PAEC à cet égard se situent à d'autres échelles. Il s'agit notamment des intoxications aiguës ou chroniques évitées, de la conservation de la biodiversité et de la résilience climatique (Ratnadass et Deguine, 2021).

$\mathrm{Vu}$ les rapports contradictoires sur les effets des différentes pratiques en termes de risques d'infections bactériennes (e.g., paillage ou introduction de volailles dans les parcelles sur les entérobactéries telluriques, ou bordures de Vetiver sur les rongeurs réservoirs), des recherches sont encore nécessaires. De même, il faut concilier, voire rendre synergiques, dans le cadre de la PAEC, des pratiques réductrices de risques d'infection bactérienne, de type «Substitution» et «Reconception».

Notre analyse montre aussi que les conclusions tirées pour un type de pathogène, en matière d'impact des pratiques de protection des cultures sur les risques sanitaires en santé publique et vétérinaire, ne sont pas forcément généralisables. Il serait donc pertinent d'étudier ce qu'il en est avec les autres groupes d'agents infectieux en santé publique et vétérinaire, notamment parasitaires (protozoaires et vers) et fongiques (champignons et oomycètes).

\section{Références}

Andru J, Cosson JF, Caliman JP, Benoit E. 2013. Coumatetralyl resistance of Rattus tanezumi infesting oil palm plantations in Indonesia. Ecotoxicology 22: 377-396. https://doi.org/10.1007/ s10646-012-1032-y.

Bessou C, Verwilghen A, Beaudoin-Ollivier L, Marichal R, Ollivier J, Baron V, et al. 2017. Agroecological practices in oil palm plantations: Examples from the field. OCL 24: D305. https://doi. org/10.1051/ocl/2017024.

Bicudo JR, Goyal SM. 2003. Pathogens and manure management systems: A review. Environmental Technology 24: 115-130. https://doi.org/10.1080/09593330309385542.

Bodey GP, Bolivar R, Fainstein V, Jadeja L. 1983. Infections caused by Pseudomonas aeruginosa. Reviews of Infectious Diseases 5: 279-313. https://doi.org/10.1093/clinids/5.2.279.

Callahan MT, Micallef SA. 2019. Waxing and cultivar affect Salmonella enterica persistence on cucumber (Cucumis sativus L.) fruit. International Journal of Food Microbiology 310: 108359. https://doi.org/10.1016/j.ijfoodmicro.2019.108359.

Chanvatik S, Donnua S, Lekagul A, Kaewkhankhaeng W, Vongmongkol V, Athipunyakom P, et al. 2019. Antibiotic use in mandarin production (Citrus reticulata Blanco) in major mandarinproducing areas in Thailand: A survey assessment. PLoS ONE 14: e0225172. https://doi.org/10.1371/journal.pone.0225172.

Couillerot O, Prigent-Combaret C, Caballero-Mellado J, MoënneLoccoz Y. 2009. Pseudomonas fluorescens and closely-related fluorescent pseudomonads as biocontrol agents of soil-borne phytopathogens. Letters in Applied Microbiology 48: 505-512. https://doi.org/10.1111/j.1472-765X.2009.02566.x.

Damasceno CL, Duarte EAA, dos Santos LBPR, de Oliveira TAS, de Jesus FN, de Oliveira LM, et al. 2019. Postharvest biocontrol of anthracnose in bananas by endophytic and soil rhizosphere bacteria associated with sisal (Agave sisalana) in Brazil. Biological Control 137: 104016. https://doi.org/10.1016/j.biocontrol.2019.104016.

Deguine JP, Gloanec C, Laurent P, Ratnadass A, Aubertot JN. 2016. Protection agroécologique des cultures. Versailles (France): Ed. Quae, 288 p.

Derne BT, Fearnley EJ, Lau CL, Paynter S, Weinstein P. 2011. Biodiversity and leptospirosis risk: a case of pathogen regulation? Medical Hypotheses 77: 339-344. https://doi.org/10.1016/j. mehy.2011.05.009.

Diaz-Sanchez S, D’Souza D, Biswas D, Hanning I. 2015. Botanical alternatives to antibiotics for use in organic poultry production. Poultry Science 94: 1419-1430. https://doi.org/10.3382/ps/pev014.

Eberl L, Vandamme P. 2016. Members of the genus Burkholderia: Good and bad guys. F1000 Research 2016 5: 1007. https://doi.org/ 10.12688/f1000research.8221.1.

Fernández M, Porcel M, de laTorre J, Molina-Henares MA, Daddaoua A, Llamas MA, et al. 2015. Analysis of the pathogenic potential of nosocomial Pseudomonas putida strains. Frontiers in Microbiology 6: 871. https://doi.org/10.3389/fmicb.2015.00871.

Gondard M, Cabezas-Cruz A, Charles RA, Vayssier-Taussat M, Albina E, Moutailler S. 2017. Ticks and tick-borne pathogens of the Caribbean: Current understanding and future directions for more comprehensive surveillance. Frontiers in Cellular and Infection Microbiology 7: 490. https://doi.org/10.3389/fcimb.2017.00490.

Grimshaw RG, Faiz A. 1995. Vetiver grass: Application for stabilization of structures. In: Proceedings of the Sixth International Conference on Low-Volume Roads, June 25-29, 1995, Minneapolis, MN, pp. 74-81.

$\mathrm{Gu}$ YH, Mazzola M. 2003. Modification of fluorescent pseudomonad community and control of apple replant disease induced in a wheat cultivar-specific manner. Applied Soil Ecology 24: 57-72. https:// doi.org/10.1016/S0929-1393(03)00066-0.

Henriquez T, Lenzi A, Baldi A, Marvasi M. 2020. Frontiers in plant breeding: Perspectives for the selection of vegetables less susceptible to enteric pathogens. Frontiers in Microbiology 11: 1087. https://doi.org/10.3389/fmicb.2020.01087.

Herth A. 2011. Le biocontrôle pour la protection des cultures. 15 recommandations pour soutenir les technologies vertes. Rapport au Premier Ministre François Fillon. https://agriculture.gouv.fr/sites/ minagri/files/documents/pdf/le_biocontrole_Rapport_Herth_web_ mail.pdf.

Hill SB, MacRae RJ. 1996. Conceptual framework for the transition from conventional to sustainable agriculture. Journal of Sustainable Agriculture 7: 81-87. https://doi.org/10.1300/J064v07n01_07.

Howell PE, Delgado ML, Scribner KT. 2017. Landscape genetic analysis of co-distributed white-footed mice (Peromyscus leucopus) and prairie deer mice (Peromyscus maniculatus bairdii) in an agroecosystem. Journal of Mammalogy 98: 793-803. https://doi.org/ 10.1093/jmammal/gyx042.

Htwe NM, Singleton GR, Johnson DE. 2019. Interaction between rodents and weeds in a lowland rice agro-ecosystem in Myanmar: The need for an integrated approach to management. Integrative Zoology 14: 396-409. https://doi.org/10.1111/1749-4877.12395.

Jechalke S, Heuer H, Siemens J, Amelung W, Smalla K. 2014. Fate and effects of veterinary antibiotics in soil. Trends in Microbiology 22: 536-545. https://doi.org/10.1016/j.tim.2014.05.005.

Khan ZR, Midega CAO, Pittchar J, Pickett JA, Bruce T. 2011 Pushpull technology: A conservation agriculture approach for integrated 
management of insect pests, weeds and soil health in Africa. International Journal of Agricultural Sustainability 9: 162-170. https://doi.org/10.3763/ijas.2010.0558.

Kumar R, Klafke GM, Miller RJ. 2020. Voltage-gated sodium channel gene mutations and pyrethroid resistance in Rhipicephalus microplus. Ticks and Tick-borne Diseases 11: 101404. https:// doi.org/10.1016/j.ttbdis.2020.101404.

Leggett MA. 2012. Managing populations of the Australasian harrier (Circus approximans) to reduce passerine bird damage in vineyards. Unpublished MSc Thesis, Lincoln University, New Zealand, $121 \mathrm{p}$.

Lenzi A, Marvasi M., Baldi A. 2021. Agronomic practices to limit pre-and post-harvest contamination and proliferation of human pathogenic Enterobacteriaceae in vegetable produce. Food Control 119: 107486. https://doi.org/10.1016/j.foodcont.2020.107486.

Litterick AM, Harrier L, Wallace P, Watson CA, Wood M. 2004. The role of uncomposted materials, composts, manures, and compost extracts in reducing pest and disease incidence and severity in sustainable temperate agricultural and horticultural crop production-A review. Critical Reviews in Plant Sciences 23: 453-479. https://doi.org/10.1080/07352680490886815.

LoGiudice K, Ostfeld RS, Schmidt KA, Keesing F. 2003. The ecology of infectious disease: Effects of host diversity and community composition on Lyme disease risk. PNAS 100: 567-571. https://doi. org/10.1073/pnas.0233733100.

Mansuy A, Fontaine R. 2014. Fiche phytosanitaire canne à sucre-Rats. Ecophytopic. http://www.bsv-reunion.fr/wp-content/ uploads/2015/02/Fiche-phyto-rats.pdf.

Marquez A, Khalil RA, Fourel I, Ovarbury T, Pinot A, Rosine A, et al. 2019. Resistance to anticoagulant rodenticides in Martinique could lead to inefficient rodent control in a context of endemic leptospirosis. Scientific Reports 9: 13491. https://doi.org/ 10.1038/s41598-019-49661-5.

Massawe AW, Rwamugira W, Leirs H, Makundi RH, Mulungu LS. 2005. Influence of land preparation methods and vegetation cover on population abundance of Mastomys natalensis in Morogoro, Tanzania. Belgian Journal of Zoology 135(Suppl): 187-190.

McCauley DJ, Salkeld DJ, Young HS, Makundi R, Dirzo R, Eckerlin RP, et al. 2015. Effects of land use on plague (Yersinia pestis) activity in rodents in Tanzania. American Journal of Tropical Medicine and Hygiene 92: 776-783. https://doi.org/10.4269/ajtmh.14-0504.

McKenna M. 2019. Antibiotics set to flood Florida's troubled orange orchards. Nature 567: 302-303. https://doi.org/10.1038/ d41586-019-00878-4.

McManus PS. 2014. Does a drop in the bucket make a splash? Assessing the impact of antibiotic use on plants. Current Opinion in Microbiology 19: 76-82. https://doi.org/10.1016/j.mib.2014.05.013.

McManus PS, Stockwell VO, Sundin GW, Jones AL. 2002. Antibiotic use in plant agriculture. Annual Review of Phytopathology 40: 443465. https://doi.org/10.1146/annurev.phyto.40.120301.093927.

Meerburg BG, Singleton GR, Kijlstra A. 2009. Rodent-borne diseases and their risks for public health. Critical Reviews in Microbiology 35: 221-270. https://doi.org/10.1080/10408410902989837.

Mendes R, Garbeva P, Raaijmakers JM. 2013. The rhizosphere microbiome: Significance of plant beneficial plant pathogenic and human pathogenic microorganisms. FEMS Microbiology Reviews 37: 634-663. https://doi.org/10.1111/1574-6976.12028.

Miarinjara A, Rahelinirina S, Razafimahatratra NL, Girod R, Rajerison M, Boyer S. 2019. Field assessment of insecticide dusting and bait station treatment impact against rodent flea and house flea species in the Madagascar plague context. PLoS
Neglected Tropical Diseases 13: e0007604. https://doi.org/ 10.1371/journal.pntd.0007604.

Nibouche S, Tibère R. 2010. Mechanism of resistance to the spotted stalk borer, Chilo sacchariphagus, in the sugar cane cultivar R 570. Entomologia Experimentalis et Applicata 135: 308-314. https:// doi.org/10.1111/j.1570-7458.2010.00996.x.

Ottesen AR, Gorham S, Pettengill JB, Rideout S, Evans P, Brown E. 2015. The impact of systemic and copper pesticide applications on the phyllosphere microflora of tomatoes. Journal of the Science of Food and Agriculture 95: 1116-1125. https://doi.org/10.1002/jsfa.7010.

Pires AFA, Patterson L, Kukielka EA, Aminabadi P, Navarro-Gonzalez N, Jay-Russell MT. 2019. Prevalence and risk factors associated with Campylobacter spp. and Salmonella enterica in livestock raised on diversified small-scale farms in California. Epidemiology and Infection 147: e321. https://doi.org/10.1017/S095026881900205X.

Polack B, Boulouis HJ, Guillot J, Chermette R. 2015. Les zoonoses (tableaux synthétiques: animaux réservoirs de pathogènes et modes de transmission). Revue Francophone des Laboratoires 477: 67-79. https://doi.org/10.1016/S1773-035X(15)30318-X.

Quénéhervé P, Dao JC, Ducelier D, Langlais C, Lassoudière A, Lhoste $\mathrm{P}$, et al. 2005. Faisabilité technique de l'agriculture biologique en Martinique : productions. In : François M, Moreau R, Sylvander B, eds. Agriculture biologique en Martinique. Montpellier (France): IRD Éditions, pp. 149-233. https://doi.org/ 10.4000/books.irdeditions. 2814.

Rahelinirina S, Duplantier JM. 1997. Suivi mensuel de la dynamique des populations de rats noirs (Rattus rattus) dans les foyers de peste de la région de Mandoto. In: Direction de la protection des végétaux $(D P V)$, Rongeurs et lutte antimurine à Madagascar, recueil des exposés lors du symposium tenu à Antananarivo du $1^{\text {er }}$ au 5 décembre, tome ii, pp.91-107.

Rahelinirina S, Duplantier JM., Ratovonjato J, Ramilijaona O, Ratsimba M, Rahalison L. 2010. Study on the movement of Rattus rattus and evaluation of the plague dispersion in Madagascar. Vector-Borne and Zoonotic Diseases 10: 77-84. https://doi.org/ 10.1089/vbz.2009.0019.

Rahelinirina S, Scobie K, Ramasindrazana B, Andrianaivoarimanana V, Rasoamalala F, Randriantseheno LN, et al. 2021. Rodent control to fight plague: Field assessment of methods based on rat density reduction. Integrative Zoology 0: 1-18. https://doi.org/10.1111/ 1749-4877.12529.

Rakotonanahary RJL, Harrison A, Maina AN, Jiang J, Richards AL, Rajerison M, et al. 2017. Molecular and serological evidence of flea-associated typhus group and spotted fever group rickettsial infections in Madagascar. Parasites \& Vectors 10: 125. https://doi. org/10.1186/s13071-017-2061-4.

Rasamiravaka T, Raveloson PA, Rajaonarivelo PJ, Rabemanantsoa C, Andrianarisoa B, Duez P, et al. 2018. Malagasy traditional treatments of infectious plant diseases exert anti-virulence activities against Pseudomonas aeruginosa and Ralstonia solanacearum. Journal of Microbiology, Biotechnology and Food Sciences 7: 377-382. https:// doi.org/10.15414/jmbfs.2018.7.4.377-382.

Ratnadass A, Deguine JP. 2021. Crop protection practices and viral zoonotic risks within a One Health framework. Science of the Total Environment 774: 145172. https://doi.org/10.1016/j.scito tenv.2021.145172.

Rodríguez-Pastor R, Escudero R, Vidal D, Mougeot F, Arroyo B, Lambin X, et al. 2017. Density-dependent prevalence of Francisella tularensis in fluctuating vole populations, Northwestern Spain. Emerging Infectious Diseases 23(8): 1377-1379. https://doi.org/10.3201/eid2308.161194. 
Rugalema G, Mnyone L. 2020. Indiscriminate use of agro-veterinary pesticides in plague endemic foci in Tanzania: Potential risk for development of insecticide resistance in flea vectors. Research Square. https://doi.org/10.21203/rs.3.rs-36506/v1.

Sellers LA, Long RF, Jay-Russell MT, Li X, Atwill ER, Engeman RM, et al. 2018. Impact of field-edge habitat on mammalian wildlife abundance, distribution, and vectored foodborne pathogens in adjacent crops. Crop Protection 108: 1-11. https://doi.org/10.1016/j.cro pro.2018.02.005.

Stockwell VO, Duffy B. 2012. Use of antibiotics in plant agriculture. Revue scientifique et technique-Office international des epizooties 31(1): 199-210. https://doi.org/10.20506/rst.31.1.2104.

Sundin GW, Castiblanco LF, Yuan X, Zeng Q, Yang CH. 2016. Bacterial disease management: Challenges, experience, innovation, and future prospects. Molecular Plant Pathology 17: 15061518. https://doi.org/10.1111/mpp.12436.

Tangkanakul W, Smits HL, Jatanasen S, Ashford DA. 2005. Leptospirosis: An emerging health problem in Thailand. Southeast Asian Journal of Tropical Medicine and Public Health 36: 281-288.

Theofel CG, Williams TR, Gutierrez E, Davidson GR, Jay-Russell M, Harris LJ. 2020. Microorganisms move a short distance into an almond orchard from an adjacent upwind poultry operation. Applied and Environmental Microbiology 86: e00573-20. https:// doi.org/10.1128/AEM.00573-20.
Tobin ME. 1994. Polynesian rats. The Handbook: Prevention and Control of Wildlife Damage. 7. https://digitalcommons.unl.edu/ icwdmhandbook/7.

van den Berg J. 2006. Vetiver grass (Vetiveria zizanioides (L.) Nash) as trap plant for Chilo partellus (Swinhoe) (Lepidoptera: Pyralidae) and Busseola fusca (Fuller) (Lepidoptera: Noctuidae). Annales de la Société Entomologique de France 42: 449-454. https://doi.org/ 10.1080/00379271.2006.10697478.

Wu S, Nishihara M, Kawasaki Y, Yokoyama A, Matsuura K, Koga T, et al. 2009. Inactivation of Escherichia coli in soil by solarization. Soil Science \& Plant Nutrition 55: 258-263. https://doi.org/ 10.1111/j.1747-0765.2009.00362.x.

Xie WY, Shen QR, Zhao FJ. 2018. Antibiotics and antibiotic-resistance from animal manures to soil: A review. European Journal of Soil Science 69: 181-195. https://doi.org/10.1111/ejss.12494.

$\mathrm{Xu}$ A, Buchanan RL, Micallef SA. 2016. Impact of mulches and growing season on indicator bacteria survival during lettuce cultivation. International Journal of Food Microbiology 224: 28 39. https://doi.org/10.1016/j.ijfoodmicro.2016.02.013.

Yoshizawa N, Usui M, Fukuda A, Asai T, Higuchi H, Okamoto E, et al. 2020. Manure compost is a potential source of tetracycline-resistant Escherichia coli and tetracycline resistance genes in Japanese farms. Antibiotics 9: 76. https://doi.org/10.3390/antibiotics9020076.

Citation de l'article : Ratnadass A, Deberdt P. 2021. Pratiques de protection des cultures en agroécosystèmes tropicaux et risques de maladies humaines et animales d'origine bactérienne. Cah. Agric. 30: 42. 